\title{
Study on the Fault Tolerance Control Strategy of the Distributed Driving Electric Vehicle Running in Straight Line with Failure Motor(s)

\author{
LIU Xinlei ${ }^{1,2, a,{ }^{*}}$ HE Hongwen ${ }^{1, b}$ XIONG Rui ${ }^{1, c}$ Tong Qian $^{1, d}$
}

1 National Engineering Laboratory for Electric Vehicles, Beijing Institute of Technology, Beijing,100081; 2 Shandong Jiaotong University, Department of Automotive Engineering, Jinan,250023. Email:liuxinlei1981@yahoo.cn.

Keywords: Distributed driving electric vehicles; straight running; fault tolerance; control strategy; in-wheel motor.

\begin{abstract}
The problem of motor fault tolerance of the distributed driving electric vehicle running in straight line is discussed and solved in the paper. The researched vehicle is designed with four in-wheel motors. The motor fault types are divided more detailed. To achieve a good riding stability and dynamic property with motors in failure state, some motor fault tolerance control strategies are proposed based on the performance of the distributed driving electric vehicles. And their mathematic models are established and simulated by MATLAB/Simulink software. The results indicate that the control strategies for torque re-allocation with motor(s) in failure state can achieve the designed demands well, and the dynamic performance of the distributed driving electric vehicle has been enhanced.
\end{abstract}

\section{Introduction}

In recent years, the electric vehicles which have advantages over traditional vehicles in saving energy and protecting environment have become one of the important research objects in many countries because of the shortage of energy and the deterioration of the environment on the earth. The distributed driving electric vehicles have created huge space for the revolution of the vehicles' structure, because their transmission chains are short, efficiencies of transmissions are high and the structures are compact. Moreover, the distributed driving electric vehicles are able to achieve some high-performance control functions which can improve the energy utilization and running performance. Therefore, the distributed driving electric vehicles which have combined energy conservation, environmental protection and safety in one, represent the future electric vehicle' important development direction. [1,2]

However, the distributed driving electric vehicles mostly use more than two motors to work independently and simultaneously. The vehicles will yaw and lose the stability if one or some motors become failure, and they probably kill the driver and passengers. So it is necessary to design the treatment mechanism for the motor faults to improve the fault tolerance ability of the vehicle controller.

At present, the study on the motor fault tolerance control of the distributed driving electric vehicles is much seldom at home and abroad. Reference [3] has studied the propeller fault tolerance control of submarine and proposed the idea which solved the system fault problem based on pseudo-inverse. The references of [4,5] used the pseudo-inverse to solve the self-repairing flight control problem of planes. Wang Bo classified fault modes of the four wheel independent drive electric vehicle and proposed the driving force distribution algorithm based on control allocation method in the references of [6]. And the control strategy of one motor fault mode was simulated by him, but it was not comprehensive and detailed. 


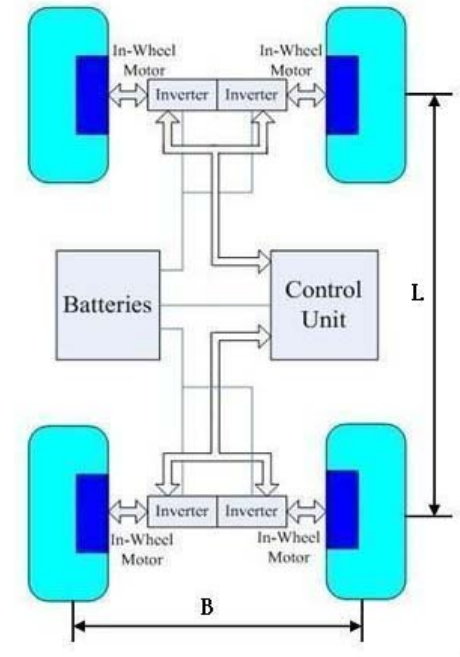

Figure 1. Schematic diagram of electric vehicle

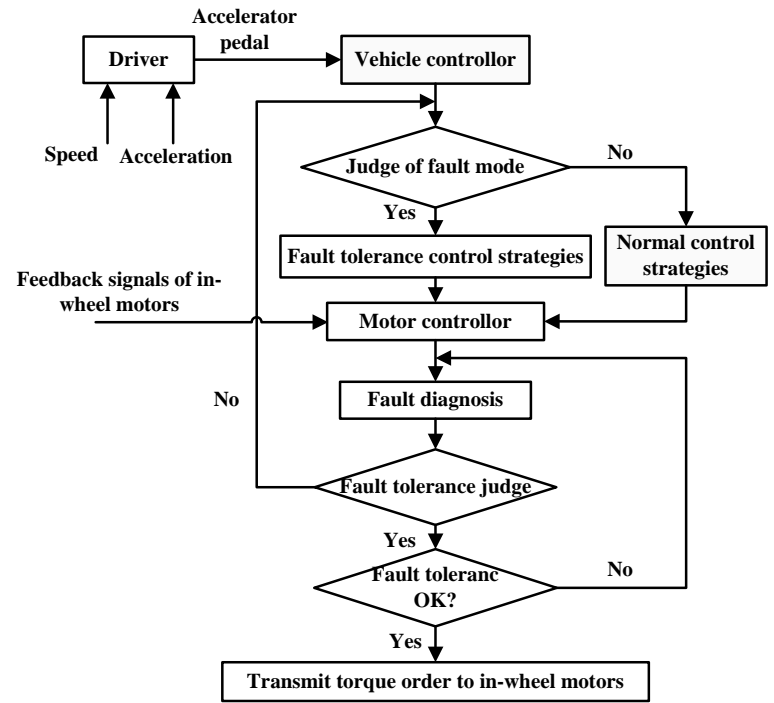

Figure.2 The flowchart of motors fault tolerance control

The study object of this paper is a distributed driving electric vehicle with four in-wheel motors as shown in the Fig.1. During running process, in-wheel motors probably have faults which can be classified into two types. The first type is part faults which do not need to stop the motors' working. However, as it can confine the working condition, the output torque of the faulty motor has constraint, for example, $T_{\text {out }}<=T_{\mathrm{s}}$. $\mathrm{T}_{\text {out }}$ is the output torque of the motor, $\mathrm{T}_{\mathrm{s}}$ is the limit torque when the motor has fault. The wheel with the fault motor(s) is still a driving wheel. Another type is full faults which can cause the motor become invalid. The wheel with the full fault motor will become a driving wheel. [7]

This paper mainly discusses tolerance control strategies for the motors' full faults. In order to reduce the level of difficulty, we assume that as follow: (1) The wheels of the research vehicle can not slip; (2) The in-wheel motors are same. (3) The vehicle is running in the straight line. This paper only discusses the vehicle's tolerance control strategies for the full fault of the in-wheel motors but the motors' tolerance control strategies for themselves. It discusses the motor fault types more detailed and studies the vehicle's tolerance control strategies further.

\section{Fault Tolerance Control System}

Fault tolerance control system is shown in Fig.2. During running of the vehicle, the driver treads the acceleration pedal according to the acceleration and speed. If some in-wheel motor gets faults, the controller of the motor will handle the faults according to the signals which supplied by the modules of monitor and diagnosis. If the faults can not be tolerated by the motor, the fault signals will be transmitted by CAN bus to the vehicle controller to judge the mode according to the signals. And then the torque will be re-allocated to the other normal in-wheel motors.

\section{Establishment of the Mathematic Model and Constraints}

The dynamical mathematic model of the vehicle is established [8]:

$$
M \dot{v}_{x}=\left(T_{1}+T_{2}+T_{3}+T_{4}\right) / r-F_{f}-F_{w}-F_{i}
$$

$M$ is mass of the vehicle; $T_{i}$ is the output torque of the in-wheel motor; the subscript " $i$ " indicates left-front wheel, right-front wheel, left-rear wheel, right-rear wheel respectively $(i=1,2,3,4) ; F_{f}$ is rolling resistance force; $\mathrm{F}_{\mathrm{w}}$ is wind resistance; $\mathrm{F}_{\mathrm{i}}$ is Climbing resistance.

The failure motor torque can be constrained by the failure factor as illustrated in the formula (2).[6] Moreover, moments of drive forces of four wheels to the centre need to be balanced after breaking down of the left-front wheel in order to avoid running deviation. So there is another constraint (3). 


$$
\begin{aligned}
& T_{i}=\beta_{i} T_{A i} \leq F_{\varphi} \cdot r \\
& \frac{T_{2}}{r} \cdot \frac{B}{2} \cdot \beta_{2}+\frac{T_{4}}{r} \cdot \frac{B}{2} \cdot \beta_{4}-\frac{T_{1}}{r} \cdot \frac{B}{2} \cdot \beta_{1}-\frac{T_{3}}{r} \cdot \frac{B}{2} \cdot \beta_{3}=0
\end{aligned}
$$

$\beta_{\mathrm{i}}$ is failure factor of the in-wheel motor $\mathrm{i}\left(\beta_{\mathrm{i}}=0,1\right) ; \mathrm{T}_{\mathrm{A}}$ is the expected torque. $\mathrm{F}_{\varphi}$ is adhesive force. $r$ is radius of vehicle's wheel.

\section{Fault Modes and Analysis to the Fault Tolerance Control Strategies}

The motor fault modes of the vehicle can be classified into three types: one motor gets fault, two motors get fault, three motors get fault. And the three types can be classified further into 15 modes, as shown in the table1.

\begin{tabular}{|c|c|c|c|c|c|c|c|c|c|}
\hline$\beta$ & $\beta_{1}$ & $\beta_{2}$ & $\beta_{3}$ & $\beta_{4}$ & ${ }^{\beta}$ & & & & \\
\hline 1 & 1 & 1 & 1 & 1 & 9 & 1 & 0 & 0 & 1 \\
\hline 2 & 1 & 1 & 1 & 0 & 10 & 0 & 1 & 0 & 1 \\
\hline 3 & 1 & 1 & 0 & 1 & 11 & 0 & 0 & 1 & 1 \\
\hline 4 & 1 & 0 & 1 & 1 & 12 & 1 & 0 & 0 & 0 \\
\hline 5 & 0 & 1 & 1 & 1 & 13 & 0 & 1 & 0 & 0 \\
\hline 6 & 1 & 1 & 0 & 0 & 14 & 0 & 0 & 1 & 0 \\
\hline 7 & 1 & 0 & 1 & 0 & 15 & 0 & 0 & 0 & 1 \\
\hline 8 & 0 & 1 & 1 & 0 & & & & & \\
\hline
\end{tabular}

Table 1. All modes of fault motors

These fifteen modes can also be classified into two cases. The first case is an un-adjustable case that the torque doesn't need to be re-allocated because that it is impossible for the vehicle to keep stability. Mode 7, mode 10, mode 12, mode 13, mode 14, mode 15 and mode 16 all belong to the un-adjustable case. If vehicle is running in these modes, it must stop immediately and needs to be repaired. Otherwise, it is easy to have an accident. Other modes belong to adjustable case; we can adopt fault tolerance control strategies to keep the normal driving in the straight line.

There are three basic principles for the motor fault tolerance control strategies. First, the stability of running in straight line must be ensured. Second, the entire drive force after torque re-allocation should be equal to the one before the motor fault happening as much as possible. Third, the economical efficiency of the motors should be optimal.

The single motor fault tolerance control strategy is much complicated. But the double-motor and multiple-motor fault tolerance control strategies which can directly allocate the torque to normal motors are much simpler than single motor's. For example, if two motors break down, the torque of the other motors will be allocated equally. But the modes of this type are more than other types. In the single motor fault tolarance control strategies, the ratio of torque of two normal motors on the other side of the fault motor can be designed off line. The ratio can also be received by on-line and on-real time optimization which takes the economy as the optimization goal.[6]

The optimization problem is described by mathematics as:

$$
\left\{\begin{array}{c}
\min \quad f(T)=\frac{T_{D N 1}}{\eta\left(n, T_{D N 1}\right)}+\frac{T_{D N 2}}{\eta\left(n, T_{D N 2}\right)} \\
\text { s.t. } T_{D N 1}+T_{D N 2}=\min \left(\frac{T_{a}}{2}, T_{\max }\right) \\
T_{D N 1} \geq 0, T_{D N 2} \geq 0 ; 1>\eta>0 ; n>0 .
\end{array}\right.
$$

The output torque of the normal motor which is on the same side of the fault motor is:

$$
T_{S N}=T_{D N 1}+T_{D N 2}
$$


$\mathrm{T}_{\mathrm{DN} 1}$ and $\mathrm{T}_{\mathrm{DN} 2}$ are the output torque of the motors which are on the opposite side of the fault motor. $\eta$ is the efficiency of motor. $n$ is rotation speed of motor. $T_{A}$ is the aim total torque which is analyzed from the acceleration pedal. $\mathrm{T}_{\max }$ is the maximum output torque of in-wheel motor. $\mathrm{T}_{\mathrm{SN}}$ is the output torque of the motor which is on the same side of the fault motor.

Basing on these mathematic models, the torque of the three normal motors can be re-allocated after some motor breaks down. In order to optimize the value of $f(T)$, the MAP of the efficiency of in-wheel is also needed. Therefore, we did a test to the in-wheel motor using dynamometer and torque admeasuring apparatus as shown in Fig3. The MAP is shown in Fig.4.

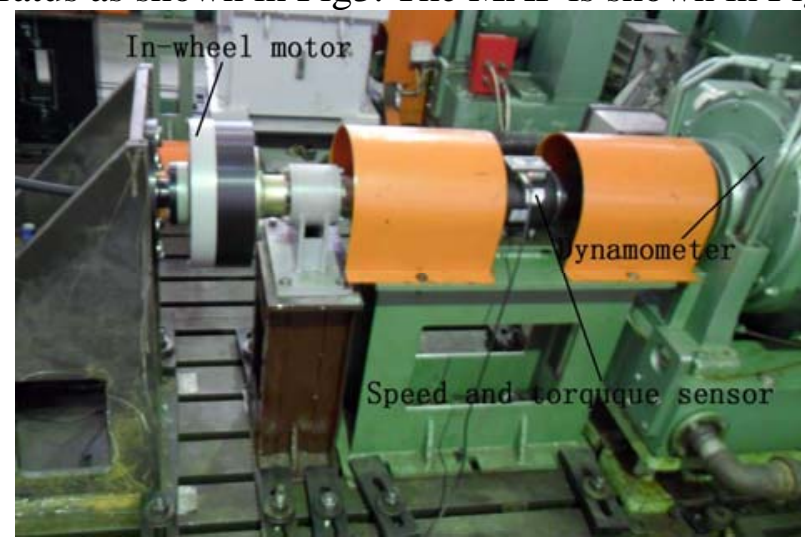

Figure.3 The test of the in-wheel motor

\section{Simulation and Analysis by a Living Example}

Table.II The Main Parameters

\begin{tabular}{clll}
\hline Parameter & Value & Parameter & Value \\
\hline Radius of wheel & $285(\mathrm{~mm})$ & Mass of vehicle & $1000(\mathrm{Kg})$ \\
Maximum torque & $140(\mathrm{Nm})$ & Rated power & $5(\mathrm{Kw})$
\end{tabular}

We assume that the test vehicle experiences full fault of single motor, double motors and three motors successively as shown in Fig.6. The vehicle runs with a uniform speed during the first 3 seconds and it needs total drive torque of $400 \mathrm{Nm}$. So every motor is allocated $100 \mathrm{Nm}$ and the fault mode is mode- 1 at the beginning. The left-front motor breaks down at the point of 3 second and the right-rear motor breaks down at the point of 13 second, too. After 10 seconds, the right-front motor breaks down at the point of 23 second. It is fault mode- 5 from the point of 3 second to 13 second, and is fault mode- 8 from the point 13 second to 23 second. At last, it is the fault mode- 14 from the point of 23 second. In the fault mode-5, the torque of the left-rear motor increases to $140 \mathrm{Nm}$ which is the maximum torque of the motor to keep the previous power performance as much as possible as shown in Fig6. In order to avoid the yawing moment, the sum of torque of the right motors is 140 $\mathrm{Nm}$, too. For the optimal economy performance, the torque of the right motors is received from optimization as shown in Fig5. $70 \mathrm{Nm}$ is the best value of them respectively.
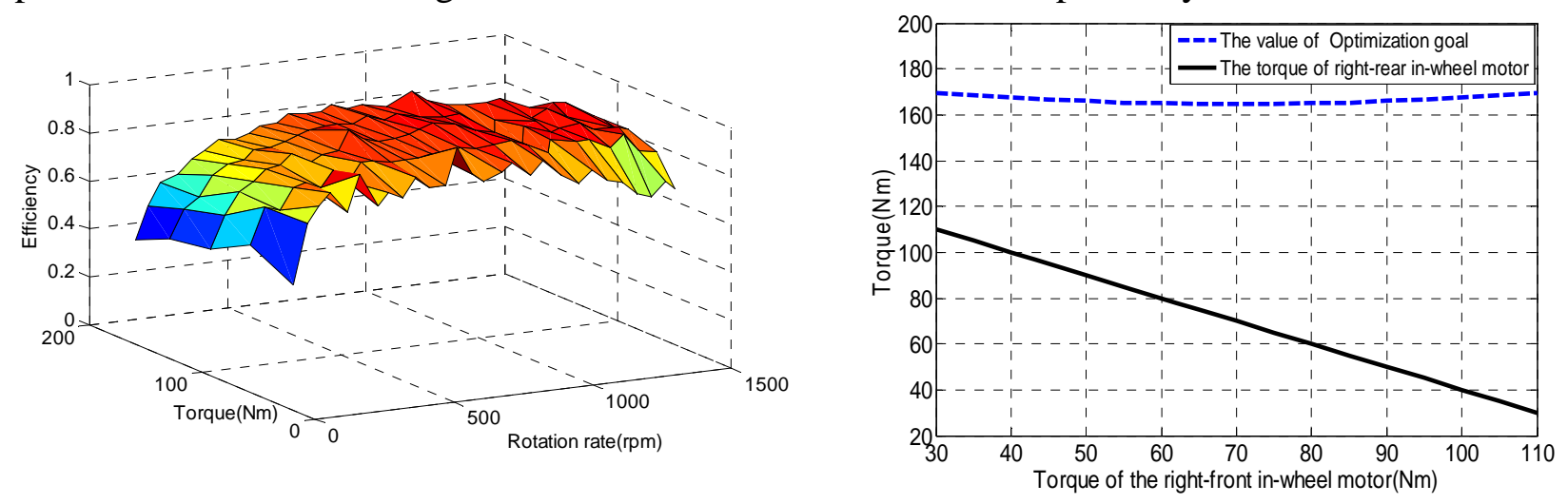
Figure.4 The MAP of efficiency of the in-wheel motor

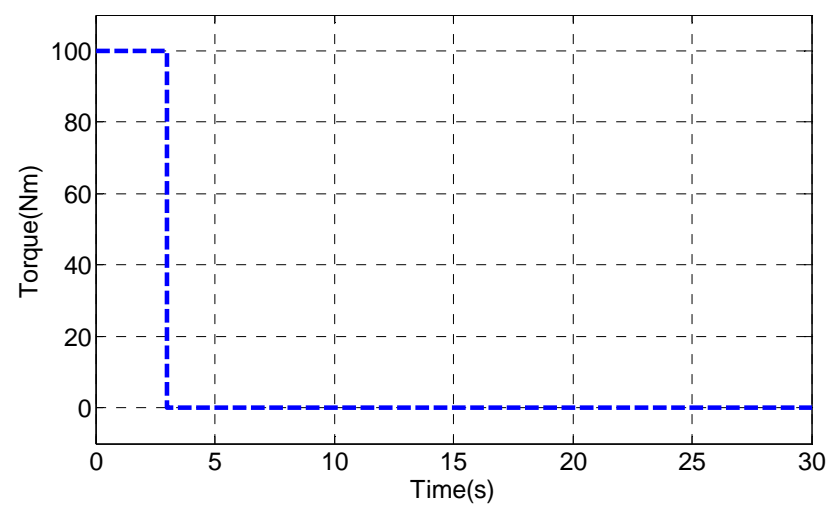

Figure.6 (a) The allocating process of toque of the left-front in-wheel motor

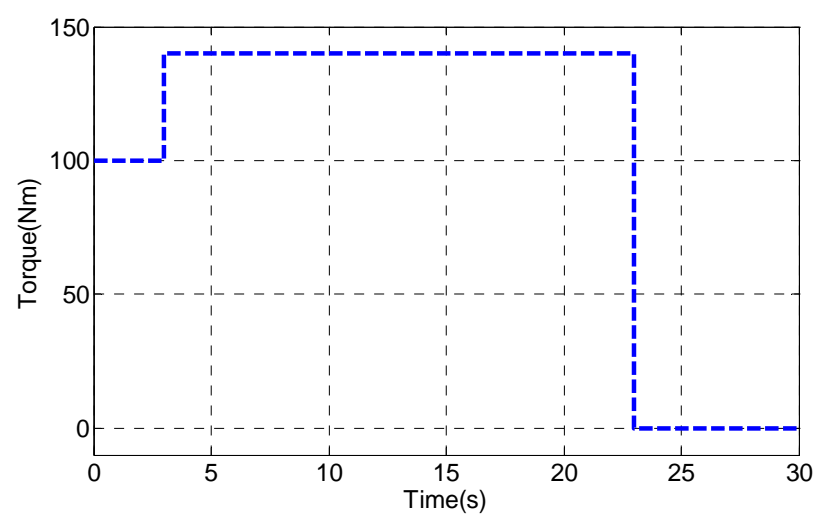

Figure.6 (c) The allocating process of toque of the left-rear in-wheel motor
Figure.5 The torque optimization curve when one motor is failure

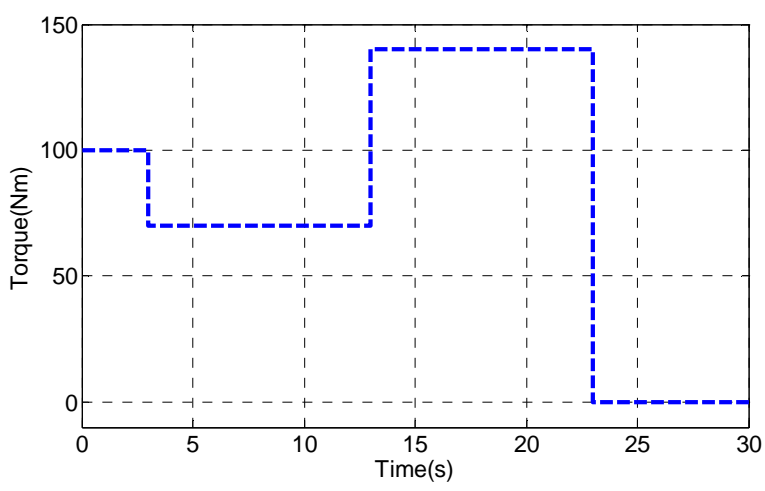

Figure.6 (b) The allocating process of toque of the right-front in-wheel motor

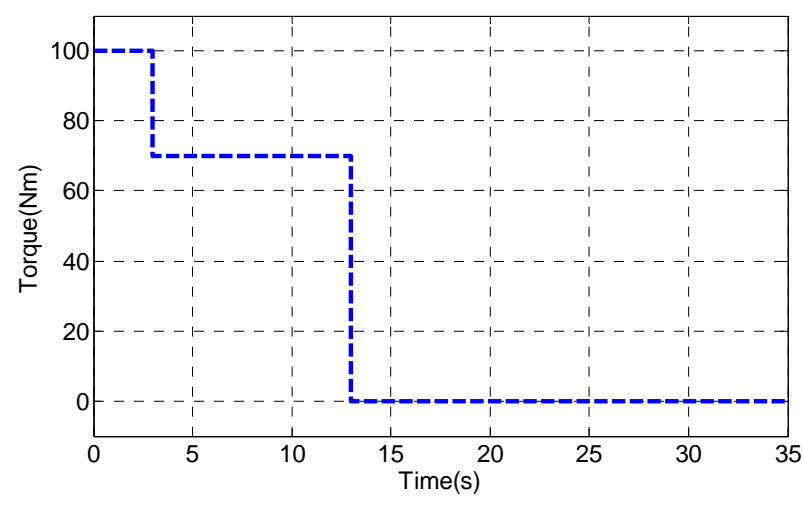

Figure.6 (d) The allocating process of toque of the right-front in-wheel motor

The torque of the left-rear motor and the torque of the left-rear motor both become to $0 \mathrm{Nm}$ in the mode-8. And the torque of the left-rear motor and the left-rear motor both become to the maximum value to keep the stability and power performance. The torque of all motors become to 0 Nm because the mode is an un-adjustable mode as shown in Fig.6.

\section{Summary and Prospect}

1. This paper introduces the classification of the motor fault of the distributed driving electric vehicle with four in-wheel motors in detail. It can be good for the recognition of mode of fault in the course of fault tolerance control. Consequently, it can offer guarantee for right tolerance control strategies easily.

2. The motor fault tolerance control strategies of the distributed driving electric vehicle with four in-wheel motors have been introduced and simulated. All these can solve the motor fault problem.

3. The motor fault tolerance control strategies of the distributed driving electric vehicle with four in-wheel motors need to be studied further. For example, when we consider the different load on the axles and the motors with different power, the problem will be more complicated.

\section{Acknowledgements}

This work was supported by the National High Technology Research and Development Program of China (2011AA112304, 2011AA11A228, 2011AA1290) in part, the International Cooperation Research Program of Chinese Ministry of Science and Technology (2011DFB70020) and the Program for New Century Excellent Talents in University (NCET-11-0785) in part.

\section{References}


[1]Yoichi Hori: Future Vehicle Driven by Electricity and Control Re-search on Four-Wheel-Motored “UOT Electric March II”[J]. IEEE Transactions on Industrial Electrics, 2004, 51(5):954-962.

[2]Kiyomoto Kawakami, et al: Development of Fail-safe Technologies of Ultra High Performance EV “KAZ”[C]. EVS18, Berlin, 2001.

[3] YU Jian-cheng, ZHANG Ai-qun, WANG Xiao-hui: Research on Thruster Fault Tolerant Control Allocation of a 7000m Manned Submarine[J].ROBOT, 2006,28(5):519-523.

[4] YANG En-quan, GAO Jin-yuan: Research and Development on Advanced Fighter Control Allocation Methods[J]. FLIGHT DYNAMICS. 2005,23(3):1-4.

[5] XIA Xiao-ye, WANG Xin-min, ZHAO Kai-rui: Application of Pseudo-Inverse Method to Self-repairing Flight Control System [J]. Fire Control \& Command Control. 2010,35(9):109-112.

[6] Wang Bo, Luo Yugong, Fan Jingjing \& Li Keqiang: A Study on Driving Force Distribution of Four-Wheel-Independent Drive Electric Vehicle Based on Control Allocation[J]. Automotive Engineering. 2010,32(2):128-132.

[7] YU Hai-fang, LIU Zhi-qiang, CUI Shu-mei: Faults and Fault Modes Analysis of Electric Vehicle Drive System in Electric vehicle [J].Power electronics. 2011.45(12):69-71.

[8] Yu Zhisheng: Automotive theory[M]. Beijing: Mechanical Engineering Press.2006(5):18-21. 